\title{
Derivation of the Strutinsky method from the least squares principle
}

\author{
B. Mohammed-Azizi ${ }^{1,2, *}$ \\ ${ }^{1}$ Laboratoire de Physique des Particules et Physique Statistique, \\ Ecole Normale Superieure de Kouba, BP 92, Vieux-Kouba, 16050 Algiers, Algeria \\ ${ }^{2}$ University of Bechar, Bechar, Algeria
}

(Dated: July 29, 2021)

The main purpose of this paper is to rigorously establish the Strutinsky method from the least squares principle. Thus, it is the mathematical basis of this method (aspect often neglected) which is revisited in an extensive way. Some formulas previously given without demonstration or in a simplified way are set out here with all the details. In this respect, the most important mathematical properties of the averaging functions are also established in this paper. When some conditions are met, it turns out that Strutinsky's method is nothing more than a polynomial moving average of the semi-classical level density.

PACS numbers: 21.60.-n, 21.10.Ma, 21.10.Dr, 02.60.-x

Keywords: Nuclear theory, Nuclear structure, Strutinsky averaging method, Binding energy

* aziziyoucef@gmail.com 


\section{INTRODUCTION}

In nuclear structure theory, the macroscopic-microscopic model is closely related to Strutinsky's discovery of the so called shell correction method [1, 2]. This model constitutes a powerful analytical tool in the study of fission barriers, equilibrium forms of nuclei and nuclear masses [3] as well as in other domains [7 9 . It continues to be applied until today [10 13, especially in extensive or systematic studies. In fact, it is to be noted that in the literature the macroscopic-microscopic method and shell correction mean the same thing. However, it should also be remembered that attempts to quantify shell correction took place before Strutinsky's discovery. In this respect, we can mention the Myers-Swiatecki version of the shell correction which was roughly defined from the notion of the energy level bunching [14].

The main strength of the macroscopic-microscopique method is that it combines a strong interaction model (the liquid drop model) with a weak interaction model (the shell model). This is due the fact that the advantages of the two models add up while their weaknesses offset each other. This association simply consists in taking the energy of the liquid drop model and in adding to it an energy correction due to the shell effects. The foundations of such an association of two very different models have been justified microscopically in Ref. [15, 16] using the Hartree-Fock method and the Fermi liquid theories.

The key element of the macroscopic-macroscopic method lies in the original idea of Strutinsky to extract a shell correction from the shell model. This mathematical procedure is not uncritical. From a practical point of view, one of the most important question is that of the plateau issue. This problem has been discussed in several references 17. 22 .

The second main criticism of Strutinsky's method lies in the fact that the function which averages (and smoothes) the quantum density of states is built only from an ansatz, i.e. from an unfounded basis. It is therefore given in a purely intuitive way. Originally, Strutinsky's idea was to replace Dirac's delta functions of the quantum density of states by Gaussians so as to get a continuous and regular level density (provided that the Gaussians are sufficiently wide). The curvature correction of the method was subsequently added because it was realized that the method was "missing something" [1. But this way of doing is more like a recipe than a rigorous scientific analysis. Later, the mathematical formalism of the Strutinky procedure evolved since the definition of smoothing will be based on a convolution product (see section IV). But even this convolution product is also given in an ad hoc way.

To remedy to this problem the present paper first proposes to demonstrate that Strutinsky level density can be precisely defined as a local polynomial smoothing of the quantum level density in the least-squares sense . In fact, this definition alone is not sufficient because the smoothing is effectively done only in a fixed window (region) of the variable. To generalize the smoothing of the level density $g(\epsilon)$ at any point $\epsilon$, one has to perform a moving average (the interval of the averaging is displaced with $\epsilon$ ). When this is is done, we recover rigorously the Strutinsky method. A moving (or rolling) average is usually a numerical technique (for discrete data) used in mathematics, statistics or in electronics, but here it is used in analytical way. The Strutinsky level density is thus rigorously established on the basis of the principle of the least squares method.

Moreover, it has been proved that within the so-called asymptotic limit, the Strutinsky density is an approximation of the semi-classical method [23. By comparing this result with the result of the present paper we can conclude that in this limit the density deduced by Strutinsky's method is a moving polynomial average of the semi-classical density.

This paper is presented as follows: In section II it is shown how to split the quantum level density into a smooth part and a rapid variations with the inherent hypotheses. Then the section III establishes the local polynomial approximation of the quantum density on the basis of the least square principle (first part of the proof ) and shows how to generalize this averaging to any point $\epsilon$ of the level density $g(\epsilon)$ by making a polynomial moving average (second part of the proof). The usual integral form of the average density of levels (which is given as a definition in most papers) is established in section IV] from the result of the previous section. Section $[\mathrm{V}$ is devoted to the main properties of the averaging function. Section VI interprets the Strutinsky method as a polynomial approach to semi-classical density in the least-squares sense. A simple example illustrating the error made by Strutinsky's method is given in section VII. The main points to remember are given in the conclusion (section VIII).

\section{SMOOTH PART OF THE QUANTUM LEVEL DENSITY}

In quantum physics, the density of states $g(\epsilon)$ is defined as the number of states per energy unit. These states are deduced from the solution of the time-independent Schrodinger equation. For a finite well, there is a finite number of bound states within the well and a continuum above. The level density is then defined as:

$$
g(\epsilon)=\sum_{i=0}^{\nu} \delta\left(\epsilon-\epsilon_{i}\right)+g_{\text {cont }}(\epsilon)
$$


For an infinite potential there are only discrete (bound) states:

$$
g(\epsilon)=\sum_{i=0}^{\infty} \delta\left(\epsilon-\epsilon_{i}\right)
$$

In this respect, it is useful to make the following remarks. From a practical point of view, the continuum is rather difficult to solve. In most of cases, the resolution of the Schrodinger equation is done through a discrete harmonic oscillator basis and all the spectrum is obtained under a discrete form. In, principle in this way, only bound states are to be considered and the continuum, obtained thus under a discrete form, is not valid and must be solved in other ways. This makes things difficult. Fortunately, there is a method 24 which avoid that, solving the level density by using also unbound states in their discrete form. In this way Eq. (1) can reduces to the more simple form given by Eq. (2). Here, in the rare cases where formula (2) is used, it will be assumed that the level density of states has been determined by the method of reference [24].

Regardless on how this function is solved, in the following, we only will assume that the function $g(\epsilon)$ is known (i.e. already solved), and that, it is not necessary to specify its explicit form.

It is also assumed that this density of states results from the superposition of a smooth monotonous function $g_{0}(\epsilon)$ and a fluctuating or oscillating part $\delta g(\epsilon)$. This can be written as:

$$
g(\epsilon)=g_{0}(\epsilon)+\delta g(\epsilon)
$$

It is known that $g_{0}(\epsilon)$ is monotonously increasing function for infinite wells whereas for finite wells it is monotonously increasing and then monotonously decreasing [24. It will be assumed that, $g(\epsilon)$ and $g_{0}(\epsilon)$ have the same asymptotic behavior for the increasing part. This is conditioned by the so called asymptotic limit [22, 23] :

$$
g(\epsilon) \sim g_{0}(\epsilon) \text { as } \epsilon \gg \hbar \omega
$$

where $\hbar \omega$ represents one quantum of the energy of the system. In fact within this limit the density of the energy levels is so large that it can be considered as continuous. This justifies the application of semi classical methods.

The aim of the proposed method is only to determine the smooth part $g_{0}(\epsilon)$ which does not contain oscillations and which can be considered as close to semi classical level density $g_{s c}(\lambda)$. As we will see, the mathematical process we are going to use, leads to the Strutinsky method.

\section{DERIVATION OF THE STRUTINSKY PROCEDURE AS A POLYNOMIAL APPROXIMATION FROM A LOCAL LEAST SQUARES PRINCIPLE}

The proof will be established in two successive steps.

\section{A. Step 1: Local least squares polynomial fit}

In a first step, we want to average or to smooth $g(\epsilon)$ by a polynomial $Q_{M}(\epsilon)$ of degree $M$, in the vicinity of some point $\epsilon=\lambda$ over a range of values weighted by a Gaussian function of effective width $\gamma$. The least squares principle will be applied by minimizing the following integral which represents a sum of squared errors:

$$
I(M, \gamma, \lambda)=\int_{-\infty}^{+\infty}\left\{g(\epsilon)-Q_{M}(\epsilon)\right\}^{2} e^{-\left(\frac{\epsilon-\lambda}{\gamma}\right)^{2}} d \epsilon
$$

Thus polynomial $Q_{M}(\epsilon)$ averages the function $g(\epsilon)$ locally over an effective range defined by the Gaussian parameter $\gamma$ around $\lambda$. By construction, the coefficients of that polynomial depend a priori on the degree $M$ (arbitrarily fixed) and because of the Gaussian, they also depend on $\gamma$ and $\lambda$. Furthermore, due to the Gaussian weight, the most interesting form is to write this polynomial as a combination of Hermite's polynomials of degree $m$ (up to $M$ ):

$$
Q_{M, \gamma, \lambda}(\epsilon)=\sum_{m=0}^{M} d_{m}(M, \gamma, \lambda) H_{m}\left(\frac{\epsilon-\lambda}{\gamma}\right)
$$


The coefficients ensuring the least squares principle will be resolved by minimization:

$$
\frac{\partial I}{\partial d_{k}}=0
$$

By Using the orthogonality property of Hermite polynomials, we finds the following coefficients:

$$
d_{k}(\gamma, \lambda)=\frac{1}{2^{k} k ! \sqrt{\pi} \gamma} \int_{-\infty}^{+\infty} g(\epsilon) H_{k}\left(\frac{\epsilon-\lambda}{\gamma}\right) e^{-\left(\frac{\epsilon-\lambda}{\gamma}\right)^{2}} d \epsilon
$$

As it can be easily seen, index $M$ does not appear in the RHS of Eq. (8), this is the reason why it has been suppressed from coefficient $d_{k}(\gamma, \lambda)$

Note: In the relationship (5), a Gaussian weight was used. In fact any weight function tending towards the Dirac delta function can be used. For example, it is perfectly possible to replace the Gaussian weight function with a Lorentz function in order to apply the least squares principle in the same way. In that respect, references [25, 26] can be consulted. In the present work, for convenience and simplicity, a Gaussian weight has been chosen. This is mainly due to the ease of use of Hermite polynomials (which are associated to the Gaussian weight).

\section{B. Step 2: Definition of the Strutinsky level density as a least squares moving average}

In fact when we want to calculate a local average of $g(\epsilon)$ at a given point $\epsilon$ we have to make the average with the values of $g(\epsilon)$ that precede and that follow the point $\epsilon$ within a given window. In our case the window is defined by the Gaussian weight which favours the values of $g(\epsilon)$ that are close to $\lambda$. Thus, due to the Gaussian weight, the averaging of $g(\epsilon)$ is inflenced mainly by the values which are near $\lambda$ (fixed in the previous step) and not by the ones that are near $\epsilon$. In order to get a true local average of $g(\epsilon)$ in which the points that precede and that follow the point $\epsilon$ play the main role, it is necessary to make the center of the bell curve (Gaussian) coincide with the point $\epsilon$ in which the average density of $g(\epsilon)$ is made. To evaluate the averaging of $g(\epsilon)$ everywhere, the bell curve (Gaussian window) must then be moved continuously with the point $\epsilon$. Averaging that function means that, at each point $\epsilon$, the value of $g(\epsilon)$ must replaced by the weighted average of its neighboring values (near $\epsilon$ ). It turns out that this process is nothing more than a moving average of function $g(\epsilon)$ or more precisely a polynomial moving average. In practice, this simply amounts to replacing straightforwardly $\epsilon$ by $\lambda$ (or conversely $\lambda$ by $\epsilon$ ) in the polynomial obtained by means of the least squares principle $(6)$ :

$$
\left.Q_{M, \gamma, \lambda}(\epsilon)\right|_{\epsilon=\lambda}=\sum_{k=0}^{M} d_{k}(\gamma, \lambda) H_{k}(0)
$$

The choice made here amounts to taking $\lambda$ (instead of $\epsilon$ ) as variable of $g$. In this case, the least square approximation of $g(\lambda)$ reads:

$$
g(\lambda) \approx Q_{M, \gamma, \lambda}(\lambda)
$$

In the following, due to the redundancy of $\lambda$ the Strutinsky density will be simply represented by $Q_{M, \gamma}(\lambda)$. It must be noted that the $Q_{M, \gamma, \lambda}(\lambda)$ polynomial approximation obtained in this way, is local, i.e. it is valid only for the point $\lambda$. For another point $\lambda^{\prime}$, the local polynomial approximation $Q_{M, \gamma, \lambda^{\prime}}\left(\lambda^{\prime}\right)$ remains valid but the two polynomials are not identical . In other words the coefficients of this polynomial change continuously with the point $\lambda$. This is essentially due the moving average introduced in the second part of the proof which makes the Strutinsky method somewhat "subtle" (see also note in section V).

In order to check that Eq. (9) give the usual well known result let us replace in Eq. (8) $g(\epsilon)$ by its value from Eq. (2), using the property of Dirac distribution, we find:

$$
d_{k}(\gamma, \lambda)=\frac{1}{2^{k} k !} \sum_{i=0}^{\infty} H_{k}\left(\frac{\epsilon_{i}-\lambda}{\gamma}\right) \frac{1}{\gamma \sqrt{\pi}} e^{-\left(\frac{\epsilon_{i}-\lambda}{\gamma}\right)^{2}}
$$

Inserting this result in Eq. (9), we obtain: 


$$
Q_{M, \gamma}(\lambda)=\sum_{i=0}^{\infty}\left\{\sum_{k=0}^{M} B_{k} H_{k}\left(x_{i}\right)\right\} \frac{1}{\gamma \sqrt{\pi}} e^{-x_{i}^{2}}
$$

with $B_{k}=H_{k}(0) / 2^{k} k$ !, and $x_{i}=\left(\epsilon_{i}-\lambda\right) / \gamma . H_{k}(0)$ is given in appendix B. We recover the usual well-known "classical" result of Strutinsky, i.e. a Gaussian multiplied by the polynomial curvature correction [6]. Thus Strutinsky density $Q_{M, \gamma}(\lambda)$ results from a polynomial moving average

The moving average defined above does not ensure that $Q_{M, \gamma}(\lambda)$ be a regular function. Thus, to smooth that quantity it is necessary to enlarge the width of the Gaussian in such way that it must be at least of the order of the average shell-spacing $\hbar \omega$ [1, 3] 5]

$$
\gamma \succsim \hbar \omega
$$

This is the well known smoothing condition of the Strutinsky procedure.

\section{DERIVATION OF THE INTEGRAL FORM OF THE MOVING AVERAGE. AVERAGING FUNCTIONS}

Coefficient $d_{k}$ is given by Eq. (8). Replacing its expression in formula (9), making $x=(\epsilon-\lambda) / \gamma$ and inverting sum and integral signs, we get an equivalent form of the smoothing average (i.e., the Strutinsky density) cited above (Eq. (9p) as follows:

$$
Q_{M, \gamma}(\lambda)=\int_{-\infty}^{+\infty} g(\lambda+\gamma x) F_{M}(x) d x
$$

where the averaging (or smoothing) function is defined by:

$$
F_{M}(x)=\left\{\sum_{m=0}^{M} B_{m} H_{m}(x)\right\} \frac{e^{-x^{2}}}{\sqrt{\pi}}
$$

with $B_{m}=H_{m}(0) / 2^{m} m$ !

There is a second form for the averaging function (see appendix C):

$$
F_{M}(x)=\left\{B_{M} \frac{H_{M+1}(x)}{2 x}\right\} \frac{e^{-x^{2}}}{\sqrt{\pi}}
$$

Moreover, an other integral form of Eq. 12 can be obtained by making again $x=(\epsilon-\lambda) / \gamma$ in that result:

$$
Q_{M, \gamma}(\lambda)=\int_{-\infty}^{+\infty} g(\epsilon) \frac{1}{\gamma} F_{M}\left(\frac{\epsilon-\lambda}{\gamma}\right) d \epsilon
$$

Here, we find the usual form of the convolution product of the Strutinsky method. In this respect, in mathematics, it is well known that there is a close relationship between the convolution product and the moving average.

In the integral form of the averaging of Eq. 115) we can replace the quantum level density by its definition from Eq. (2) Applying property of Dirac distribution one gets:

$$
Q_{M, \gamma}(\lambda)=\sum_{i=0}^{\infty} \frac{1}{\gamma} F_{M}\left(\frac{\epsilon_{i}-\lambda}{\gamma}\right)
$$

It must be noted that Eq. (16) and Eq. (10) are identical. This result is achieved in two different ways and constitutes a check of this formula. We also have to add the same smoothing condition (11) as seen before. 


\section{PROPERTIES AND WIDTH OF THE AVERAGING FUNCTION}

The basic properties of the averaging functions $F_{M}(x)$ are given in appendix A. Most of them are necessary for understanding or demonstrations The non obvious properties are demonstrated in Appendix D

The most fundamental property is the following integral transform (giving the the Strutinsky density) already seen in Eq. 15] in which $g(\epsilon)$ represents here any function (not necessarily a level density):

$$
\int_{-\infty}^{+\infty} g(\epsilon) \frac{1}{\gamma} F_{M}\left(\frac{\epsilon-\lambda}{\gamma}\right) d \epsilon=g(\lambda)+\sum_{k=M+2}^{\infty} D_{k M} \gamma^{k} \frac{d^{k} g(\lambda)}{d \lambda^{k}}
$$

In which:

$$
D_{k M}=\left(\frac{(-1)^{M / 2}}{(M / 2) !}\right)\left(\frac{1}{[(k-M-2) / 2] !}\right)\left(\frac{1}{2^{k-1} k}\right), k, M=\text { even }
$$

This formula is obtained by making a Taylor expansion of $g(\lambda+\gamma x)$ in Eq. (12) and using properties number 5, 6 and 9 of the table given in appendix $\mathrm{A}$. From this formula some consequences can be deduced:

- Because of the derivatives in Eq. (17), if $g(\epsilon)$ is a polynomial of degree $M$ (or less), the series vanishes and the integral transform of Eq. (17) gives back to the same polynomial $g(\lambda)$ (in $\lambda$ ). So if $g(\epsilon)$ is a polynomial this integral returns the same polynomial (in $\lambda$ ). Conversely, if $g(\epsilon)$ is not a polynomial, this transformation gives back again to $g(\lambda)$ but with a remainder which is of the order of the first term of the series (for which $k=M+2)$.

- If $\gamma=0$, the series in the right-hand side of Eq. (17) cancels. This means that integral in Eq. (17) gives back to the same function. In other words $\frac{1}{\gamma} F_{M}\left(\frac{\epsilon-\lambda}{\gamma}\right)$ reduces to Dirac function in the limit $\gamma \rightarrow 0$. Analogous property holds if $M \rightarrow \infty$. Indeed, in this case, the remainder of the Taylor expansion of $g(\epsilon)$ in the integral of Eq. (17) also cancels involving the same consequence for the series in Eq. (17) (which is in fact, the integral of this remainder). So, in that situation, $\frac{1}{\gamma} F_{M}\left(\frac{\epsilon-\lambda}{\gamma}\right)$ reduces also to Dirac function.

- As regard the real width of the averaging functions (see also points just below), the above properties shows that, the actual width of the function $F_{M}(x)$ is not due to the sole $\gamma$ parameter (which characterizes the width of the Gaussian) but is governed by the both parameters $\gamma$ and $M$. It increases with $\gamma$ and diminishes with $M$.

- An other notable property is that the area of this function is always normalized to the unit. This can be shown by simply making $g(\epsilon)=1$ in Eq. (17). Its main maximum (in $x=0$ ) increases when its width decreases and vice versa. Thus:

$$
\int_{-\infty}^{+\infty} \frac{1}{\gamma} F_{M}\left(\frac{\epsilon-\lambda}{\gamma}\right) d \epsilon=1
$$

On the other hand, without going into further details, one also can say that:

- Due to the Hermite polynomial in the second representation given by Eq. (14), the averaging function has $M$ symmetrical roots with respect to the $x=0$ axis, $(M / 2)$ positive and $(M / 2)$ negative with $M$ even. It has a main maximum in $x=0$ and successive oscillations with an amplitude that decreases rapidly (see Fig. 1D).

- The main contribution of this area comes from the central part because these oscillations decrease rapidly in amplitude and also because they have successive areas that are of opposite signs and thereby compensate each other. In terms of area, the curve of $F_{M}(x)$ is practically confined between -1 and +1 . The following property is all the more true as the quantity $a$ is large compared to the unit.

$$
\int_{-a}^{+a} F_{M}(x) d x \approx 1 \quad \text { if } \quad a \gg 1 \quad \forall M
$$


In effect, it is to be noted that even for $a=1$, the precision is already satisfactory. For example, taking $M=30$ and $a=1$ the value of the integral will be about 0.9914. For $M=2$ and $a=1$, one obtains 1.050. Because the oscillations change sign, the values of the area are given either by excess or by default.

Curve $(1 / \gamma) F_{M}(x / \gamma)$ has an analog property as the one given by Eq. $[19)$, i.e. are practically confined between $-\gamma$ and $+\gamma$. In other words, it has a "quasi-width" of length about $2 \gamma$.

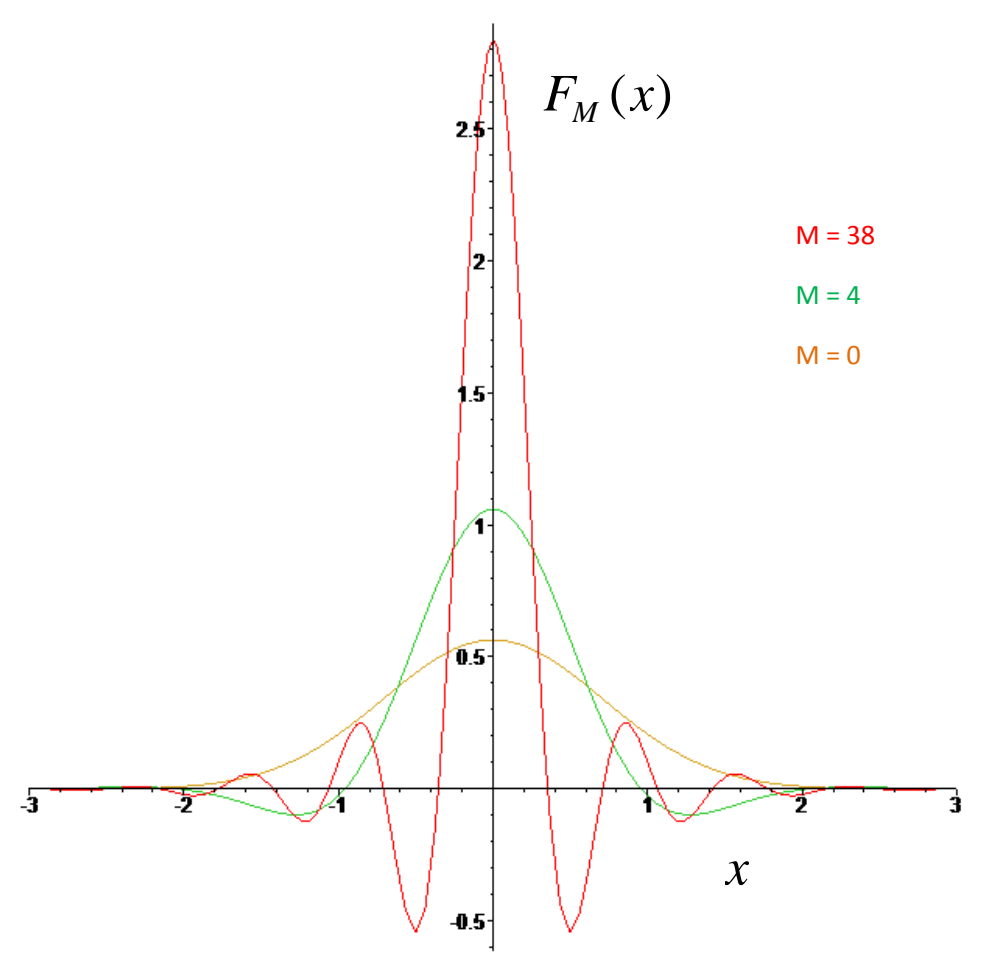

FIG. 1. Averaging functions Eq. $(\sqrt{14})$ for three values of the degree $M$. It is to be noted that these functions practically cancel as soon as $\| \gtrsim 2$

\section{THE RELATIVE REMAINDER OF THE AVERAGING IN THE ASYMPTOTIC LIMIT}

Because the right-hand side of Eq. (15) is equal to the Strutinsky density $Q_{M, \gamma}(\lambda)$, we can deduce the link between the Strutinsky level density $Q_{M, \gamma}(\lambda)$ and the quantum density $g(\lambda)$. Indeed, rewriting Eq. (17) as follows: we find that:

$$
Q_{M, \gamma}(\lambda)=g(\lambda)\left(1+R_{M+2, \gamma}(\lambda)\right)
$$

where the relative remainder is the sum of as a Taylor remainder divided by $g(\lambda)$. This relative remainder is of the order of the first element of this sum, i.e:

$$
R_{M+2, \gamma}(\lambda) \approx \frac{(-1)^{M / 2}}{(M / 2) ! 2^{M+1}(M+2)}\left(\frac{g^{(M+2)}(\lambda)}{g(\lambda)}\right) \gamma^{M+2}
$$


in which $g^{(M+2)}(x)$ represents the $(M+2) t h$ derivative ( $M$ even). Because actually this formula comes from the Taylor expansion of $g(\lambda+\gamma x)$, the Taylor remainder will be small only if condition $\lambda \gg \gamma|x|$ is well satified. Due to property 19, this condition turns to be:

$$
\lambda \gg \gamma
$$

which in fact is equivalent to the asymptotic limit defined in section II (where variable $\epsilon$ has simply been replaced by $\lambda)$ :

$$
\lambda \gg \hbar \omega
$$

because $\gamma$ and $\hbar \omega$ are of the same order. To show that, it is sufficient to note that the compatibility between condition 11 and asymptotic limit 22 can be summarized in only one formula:

$$
\lambda \gg \gamma \gtrsim \hbar \omega
$$

Classically, $\lambda$ and $\hbar \omega$ are connected to physical conditions, thus the only free parameter, i.e. $\gamma$ should be neither too large nor too small. The optimal choice is then necessarily about $\gamma \approx \hbar \omega$, in this case Eq. 22 and condition 23 . are thus equivalent.

Consequently, if conditions of Eq. 24 are fulfilled, $g(\lambda)$ will be smooth, like $g_{0}(\lambda)$ defined in section II and will be close to the semi classical density (see Ref. [23]). In addition, under these conditions, the remainder $R_{M+2}(\lambda)$ will be small. In this case, the Strutinsky level density can be considered as a local polynomial approximation to the semi classical density. This is why the Strutinsky procedure gives the exact result for $M \geq 2$ in the case of the three dimensional harmonic oscillator where the semiclassical level density is rigorously a polynomial of degre two (parabola) [25].

\section{ILLUSTRATIVE EXAMPLE OF THE ACCURACY OF THE STRUTINSKY METHOD. PARTICLE IN A CUBIC BOX WITH REFLECTING WALLS}

For the cubic box it is possible to estimate the relative error of the Strutinsky method with respect to semi classical result. For this example, the eigenvalues are:

$$
\epsilon_{n_{x} n_{y} n_{z}}=\left(n_{x}^{2}+n_{y}^{2}+n_{z}^{2}\right) E_{0}, \quad E_{0}=\frac{\pi^{2} \hbar^{2}}{2 m a^{2}}
$$

herein $n_{x}$ or $n_{y}$ or $n_{z}=1,2, \ldots \ldots$ are quantum numbers (the value 0 is forbidden because the wave function cancels in this case), $\hbar$ is the Planck constant and $m$ is the mass of the particle and $a$ the side of the box.

A Truncated asymptotic series can be obtained from Eq. (16) by using the Euler MacLaurin formula. (without derivatives and highest terms). The calculations are somewhat lengthy but simple and include expansions of binomial series that must be truncated after using property 9 of the table in appendix A For example, taking $M=8$ and $M=30$, after some algebra and some numerical evaluations, the result of the Strutinsky approximation in the case of the cubic box for $M=8$ and $M=30$ are :

$Q_{8, \gamma}(\lambda) \approx \frac{\pi \lambda^{1 / 2}}{4 E_{0}^{3 / 2}}\left(1+10^{-6}\left(\frac{\gamma}{\lambda}\right)^{10}\right)-\frac{3 \pi}{8 E_{0}}+\frac{3}{8 E_{0}^{1 / 2} \lambda^{1 / 2}}\left(1+10^{-5}\left(\frac{\gamma}{\lambda}\right)^{10}\right)$

$Q_{30, \gamma}(\lambda) \approx \frac{\pi \lambda^{1 / 2}}{4 E_{0}^{3 / 2}}\left(1+10^{-9}\left(\frac{\gamma^{\prime}}{\lambda}\right)^{32}\right)-\frac{3 \pi}{8 E_{0}}+\frac{3}{8 E_{0}^{1 / 2} \lambda^{1 / 2}}\left(1+10^{-7}\left(\frac{\gamma^{\prime}}{\lambda}\right)^{32}\right)$

For the cubic box, the semi classical result (exact solution) is well known [27]:

$g_{s c}(\lambda)=\frac{\pi \lambda^{1 / 2}}{4 E_{0}^{3 / 2}}-\frac{3 \pi}{8 E_{0}}+\frac{3}{8 E_{0}^{1 / 2} \lambda^{1 / 2}}$

Thus, the relative error is of the of the order of $10^{-6}(\gamma / \lambda)^{10}$ and $10^{-9}\left(\gamma^{\prime} / \lambda\right)^{32}$ for the cited examples. For realistic cases, the error is not so small, but it is still very acceptable. In this respect, it can be said that if Strutinsky method is applied correctly, it gives good results and thus can be considered as an alternative to semi-classical methods.

According to the remark made in the third point of section $\mathrm{V}$ in these expressions (for $M=8$ and $M=30$ ) the value of the smoothing parameter is not the same $\left(\gamma^{\prime} \neq \gamma\right)$ because this parameter increases with the degree $M$. 


\section{CONCLUSION}

The work made in this paper proves that the smoothing method early discovered by Strutinsky and used as a simple prescription can in fact be founded by imposing the least squares principle in the smoothing procedure. More specifically, the smoothing of the quantum density must be made by a local polynomial approximation (with a fixed degree) by minimizing the squared error. However, this definition is not sufficient and leads to a "static average". In order, to perform the average at any point of the level density it is necessary to make a polynomial moving average. This is the reason why the proof is done in two steps. We can summarize that by stating that the Strutinsky prescription can be rigorously established starting from a principle of "a polynomial moving average in the least squares sense". In addition, this paper demonstrates also how to obtain the integral representation of that average (known also as a convolution product). The averaging function in the integrand has been extensively studied. Practically, all the properties of the averaging function have been given.

Last but not least, it turns out that when the smoothing and asymptotic limit conditions are fulfilled, the Strutinsky method is nothing more than a polynomial moving average of the semi classical level density. Consequently, Strutinsky's method can be a good alternative to semi-classical methods.

\section{Appendix A: Main properties of the averaging (or smoothing) functions}

The main properties of the averaging (or smoothing) functions are given in the following table:

\begin{tabular}{|c|c|c|c|}
\hline & Property & Condition & \\
\hline 1 & $F_{M}(x)=0$ & $M$ odd & due to the def. of $B_{M}$ \\
\hline 2 & $F_{M}(-x)=F_{M}(x)$ & $M$ even & M even in the following \\
\hline 3 & $\int_{-\infty}^{+\infty} G(x) F_{M}(x) d x=0$ & $G(x)$ odd function & for parity reason \\
\hline 4 & $\int_{-\infty}^{+\infty} F_{M}(x) d x=1$ & & see section $\mathrm{V}$ \\
\hline 5 & $\int_{-\infty}^{+\infty} \frac{1}{\gamma} F_{M}\left(\frac{\epsilon-\lambda}{\gamma}\right) d \epsilon=1$ & & variable change in 4 \\
\hline 6 & $\int_{-\infty}^{+\infty} x^{k} F_{M}(x) d x=0$ & $1 \leq k \leq M$ & see appendix $\mathrm{D} 2$ \\
\hline 7 & $\int_{-\infty}^{+\infty} x^{k} F_{M}\left(x-x_{0}\right) d x=x_{0}^{k}$ & $1 \leq k \leq M$ & variable change in 6 \\
\hline 8 & $\int_{-\infty}^{+\infty} \epsilon^{k} \frac{1}{\gamma} F_{M}\left(\frac{\epsilon-\lambda}{\gamma}\right) d \epsilon=\lambda^{k}$ & $1 \leq k \leq M$ & variable change in 7 \\
\hline \multirow[t]{2}{*}{9} & $\int_{-\infty}^{+\infty} x^{k} F_{M}(x) d x=C_{k, M}$ & $k \geq M+2, \quad k$ even & see appendix $\mathrm{D} 2$ \\
\hline & & $C_{k, M}=\frac{(-1)^{M / 2}(k-1) !}{(M / 2) ! 2^{k-1}((k-M-2) / 2) !}$ & \\
\hline 10 & $\int_{-\infty}^{+\infty}(\epsilon-\lambda)^{k} \frac{1}{\gamma} F_{M}\left(\frac{\epsilon-\lambda}{\gamma}\right) d \epsilon=C_{k, M} \gamma^{k}$ & $k \geq M+2, \quad k$ even & variable change in 9 \\
\hline
\end{tabular}

TABLE I. Main properties of the averaging functions.

Appendix B: Hermite polynomials:

\section{Definition}

$H_{n}(x)=(-1)^{n} e^{x^{2}} \frac{d^{n}}{d x^{n}} e^{-x^{2}}$ with $n=0,1,2, \ldots$ The degree of this polynomial is $n$.

\section{Orthogonality and norm:}

$\int_{-\infty}^{+\infty} H_{n}(x) H_{m}(x) e^{-x^{2}} d x=2^{n} n ! \sqrt{\pi} \delta_{n m}$ where $\delta_{n m}$ is the Kronecker symbol.

\section{Parity and special values}

$$
\begin{aligned}
& H_{n}(-x)=(-1)^{n} H_{n}(x) \\
& H_{n}(0)=\frac{(-1)^{n} n !}{(n / 2) !} \quad n \text { even, } H_{n}(0)=0 \quad n \text { odd }
\end{aligned}
$$




\section{Well known property:}

$\int_{-\infty}^{+\infty} x^{k} H_{m}(x) e^{-x^{2}} d x=0 \quad$ for $k=0,1,2, \ldots \ldots, m-1$

In other words: $H_{m}(x)$ is orthogonal to any polynomial of degree less than $m$, with $k<m$.

\section{Appendix C: Christopher-Darboux formula applied to averaging functions}

The Christopher-Darboux formula is:

$$
\sum_{m=0}^{M} \frac{H_{m}(x) H_{m}(y)}{2^{m} m !}=\frac{1}{2} \frac{H_{M+1}(x) H_{M}(y)-H_{M+1}(y) H_{M}(x)}{2^{M} M !(x-y)}
$$

Multiplying this equality by $e^{-x^{2}} / \sqrt{\pi}$, making $y=0$, we recover $F_{M}(x)$ of Eq. 13 . On the other hand, Hermite polynomial has the property $H_{m}(0)=0$ for odd $m$. Consequently the sum contains only terms corresponding to even values $m$ up to even $M$. The result is:

$F_{M}(x)=\left\{\sum_{m=0}^{M} B_{m} H_{m}(x)\right\} \frac{e^{-x^{2}}}{\sqrt{\pi}}=\left\{B_{M} \frac{H_{M+1}(x)}{2 x}\right\} \frac{e^{-x^{2}}}{\sqrt{\pi}}$

Where the constant $B_{M}$ is defined by:

$B_{P}=\frac{(-1)^{P / 2}}{2^{P}(P / 2) !} \quad P$ even,$\quad B_{P}=0 \quad P$ odd

\section{Appendix D: Demonstrations of some Formulas:}

\section{Orthogonality between monomials and averaging functions}

This comes essentially from the property given by the property of appendix (B 4).

$\int_{-\infty}^{+\infty} x^{k} F_{M}(x) d x=0 \quad k=1,2,3, \ldots, M \quad(M=$ even $)$ In effect, using the second form of $F_{M}(x)$ given in Eq. 14$)$, we can write:

$\int_{-\infty}^{+\infty} x^{k} F_{M}(x) d x=\int_{-\infty}^{+\infty} x^{k}\left\{B_{M} \frac{H_{M+1}(x)}{2 x}\right\} \frac{e^{-x^{2}}}{\sqrt{\pi}} d x=\left(B_{M} / 2\right) \int_{-\infty}^{+\infty} x^{k-1} H_{M+1}(x) \frac{e^{-x^{2}}}{\sqrt{\pi}} d x=0$ if $k-1<M+1$.

Due to the result of appendix ( $(\mathrm{B} 4)$, the previous inequality amounts to taking $k<M+2$ or $k=2,4,6 \ldots M$.

\section{Property of Hermite polynomials}

An important property (used in this work) of Hermite polynomials is:

$\int_{-\infty}^{+\infty} x^{k} H_{m}(x) e^{-x^{2}} d x=\frac{\sqrt{\pi} k !}{2^{k-m}\left(\frac{k-m}{2}\right) !} \quad$ if $\quad k \geq m \geq 0 \mathrm{~T}$

This property has been proved from combinations of the following equation [28]:

$\int_{-\infty}^{+\infty} x^{2 w+m} H_{m}(x) e^{-x^{2}} d x=\frac{2^{m} \Gamma\left(\frac{2 w+m+1}{2}\right) \Gamma\left(\frac{2 w+m+2}{2}\right)}{\Gamma(w+1)} \quad w=0,1,2, \ldots$ with the well known property of the Gamma function

$\Gamma(z) \Gamma(z+1 / 2)=2^{1-2 z} \sqrt{\pi} \Gamma(2 z)$

and $\Gamma(n+1)=n$ !

\section{Important property of averaging function}

Appendix D2 leads to the following formula:

$\int_{-\infty}^{+\infty} x^{k} F_{M}(x) d x=C_{k, M}=\frac{(-1)^{M / 2}}{(M / 2) !} \frac{(k-1) !}{2^{k-1}} \frac{1}{((k-M-2) / 2) !} \quad k=M+2, M+4, M+6, \ldots \ldots \ldots \infty$

Using the second form of $F_{M}(x)$, i.e. Eq. (14), we obtain:

$\int_{-\infty}^{+\infty} x^{k} F_{M}(x) d x=\int_{-\infty}^{+\infty} x^{k}\left\{B_{M} \frac{H_{M+1}(x)}{2 x}\right\} \frac{e^{-x^{2}}}{\sqrt{\pi}} d x=\left(B_{M} / 2\right) \int_{-\infty}^{+\infty} x^{k-1} H_{M+1}(x) \frac{e^{-x^{2}}}{\sqrt{\pi}} d x=0 \quad$ if $k-1<M+1$.

Thus, making $k-1 \rightarrow k$ and $M+1 \rightarrow M$ in the result of appendix D 2, we then obtain the result. 
[3] M. Brack, J. Damgard, A. S. Jensen, H. C. Pauli, V. M. Strutinsky, and C. Y. Wong, Rev. Mod. Phys. 44 (1972) 320.

[4] S. G. Nilsson, C. F. Tsang, A. Sobiczewski, Z. Szymanski, S. Wycech, C. Gustafson, I. L. Lamm , P. Moller and B. Nilsson, Nucl. Phys. A $131(1969) 1$

[5] M. Bolsterli, E . O. Fiset, J. R. Nix and J. L. Norton, Phys. Rev. C 5 (1972) 1050

[6] J. R. N i x , Ann. Rev. Nucl. Sci. brack 198122 (1972) 65.

[7] S.M. Reimann, M. Brack and K. Hansen, Z. Phys. D 28 (1993) 235.

[8] D. N. Poenaru and I. H. Plonski , Romanian Reports in Physics, Vol. 60, No. 3, P. 529-538, 2008

[9] Baojing Zhou and Yan Alexander Wang, The Journal of Chemical Physics 127, 064101 (2007); doi: 10.1063/1.2755714

[10] R. A. Gherghescu and D. N. Poenaru, Pramana journal of physics, Vol. 85, No. 3, pp. 439-446 (2015)

[11] G.G. Adamian, L.A. Malov, N.V. Antonenko, H. Lenske, Kun Wang, and Shan-Gui Zhou, Eur. Phys. J. A (2018) 54: 170

[12] T.S. Fan, Z.M. Wang, X. Zhu, W.J. Zhu, and C.L. Zhong, EPJ Web of Conferences 146, 04033 (2017)

[13] Zhiming Wang, Wenjie Zhu, Chunlai Zhong, Tieshuan Fan, Nucl. Phys. A 989 (2019) 81-96

[14] W. D. Myers and W. J. Swiatecki, Nucl. Phys. 81 (1966) 1-60

[15] G. G. Bunatian,V. M. Kolomietz and V. M. Strutinsky, Nucl. Phys. A188 (1972) 225-258;

[16] M. Brack and P. Quentin, Nucl. Phys. A361 (1981) 35 - 82

[17] Wing Fai Lin, Phys. Rev. vol.2, numb. 3 (1970)

[18] V.S. Ramamurthy, M. Prakash and S.S. Kapoor, physics Letters, Vol. 62B, numb. 2 (1976)

[19] Niu Yi-Fei, Liang Hao-Zhao, Meng Jie, Chin. Phys. Lett. Vol.26, No.3 (2009) 032103

[20] P. Salamon, A. T. Kruppa, T. Vertse, Phys. Rev. C 81, 064322 (2010)

[21] K. Pomorski a and B. Nerlo-Pomorska, Eur. Phys. J. A (2016) 52: 144

[22] B. Mohammed-Azizi, Phys. Rev. C 100, 034319 (2019)

[23] B. Mohammed-Azizi and D. E. Medjadi, Phys. Rev. C 74, 054302 (2006)

[24] T. Vertse, A. T. Kruppa, and W. Nazarewicz Phys. Rev. C, 2000, v. 61, 064317

[25] M. Brack and H. C. Pauli, Nucl. Phys. A 207, 401 (1973).

[26] Alphonse P. Magnus, Viviane Pierrard, Journal of Computational and Applied Mathematics 219 (2008) 431-440

[27] R K Bhadun and C. K Ross, Phys. Rev Lett. 27 (1971) 606

[28] Sadhana Mishra, Annales Polonici Mathematici 56 (1991), 19-28. DOI: 10.4064/ap-56-1-19-28 